\title{
Modelling of cross organizational manufacturing resource service chain based on service supply-demand dynamic matching network
}

\author{
Qingqing Yang*, Jia Liu, and Kewei Yang \\ College of systems engineering, National University of Defense Technology, Changsha, China, \\ 410073
}

\begin{abstract}
In the cloud manufacturing systems, both manufacturing tasks and manufacturing services are in a dynamic environment. How could cloud manufacturing platform optimizes manufacturing cloud services based on QoS, matching an optimal service composition for manufacturing tasks has become an urgent problem at present. In view of this problem, we study the matching of manufacturing tasks and manufacturing services from the perspective of complex network theory. On the basis of manufacturing task network and manufacturing service network, a dynamic matching network theory model of manufacturing task-service is constructed. And then, we take a dynamic assessment of QoS. Finally, we use load and dynamic QoS as the optimization objectivities, transform the optimal manufacturing service composition problem into the shortest path problem, and the dynamic scheduling of manufacturing services is realized.
\end{abstract}

\section{Introduction}

Because of the diversification of customer needs, the manufacturing industry is increasingly trying to integrate products and service components together to quickly respond to customer needs, shorten product development cycles and maintain agility by creating more complete solutions. However, shifting from a product-centric perspective to a solutionoriented perspective is challenging. [1] manufacturing tasks and manufacturing services are in a dynamic and changing environment. Because of the dynamic nature of manufacturing resources, manufacturing services are in a dynamic environment, including the addition of new services, the evolution or extinction of old services. Exceptions may also occur to the services being invoked. [2] In view of dynamic and changeable operating environment, there are many different researchers study the issue of supply demand matching from different angles. Moon etc. building an integer programming model to match the supply and demand of manufacturing resource services to ensure the optimality of QoS. [3] Zeng ets put forward the idea of Global re-planning to cope with the anomalies in service composition. [4] However, re-planning will bring additional costs. For this reason, Dai et al.

\footnotetext{
* Corresponding author: qingqingyang1015@163.com
} 
proposed a self-healing method for service composition, which reduces the number of re selection through performance prediction. [5] Dotoli etc. design an integrated E-supply chain for agile and environmentally conscious manufacturing. [6] Most of these methods cannot be well applied to manufacturing services. Firstly, there is no systematic approach to considering changes in demand and services. Secondly, compared with the traditional Web service environment, the complexity and uncertainty of cloud manufacturing environment are more prominent. Thirdly, the variety of manufacturing resources is more complex and diverse. As the result, the existing researches on supply and demand matching and optimal allocation of manufacturing resources (for example, the problem of matching for simple demand or primitive task[7] and matching for complex demand or compound task[8]) are still far from the service-oriented practice.

The dynamic changes of manufacturing tasks and manufacturing services pose challenges to the management of cloud manufacturing systems. At present, there is a lack of in-depth research on the supply-demand dynamic matching method in the cloud manufacturing environment, and no mature scheme has been formed. We will use the theory of complex network to construct the service-demand dynamic matching network model. The model will provide a theoretical basis for the dynamic management of manufacturing tasks and manufacturing services.

\section{Problem description}

In service-oriented manufacturing systems, the supply is the set of various social available manufacturing resources and capabilities in the form of services.Nodes load of service manufacturing, which are denoted as L, referring to the number of manufacturing tasks currently processed by manufacturing services. Quality of Service (QoS), which refers to the non-functional attributes of manufacturing services. QoS is an important basis for the service composition.

In the traditional manufacturing service composition, we need to pick up one suitable service from the service classes of $C_{1}, C_{2}$ and $C_{3}$ to build the service composition for a manufacturing task sequence $T_{1} \rightarrow T_{2} \rightarrow T_{3}$, Each service class contains several services which having same or similar function but different in QoS, as shown in fig.1.

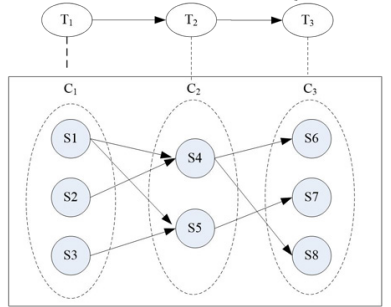

Fig.1. Problem scenario of manufacturing service composition.

Based on the potential call relationship between services, we can choose different service composition to carry out the task, e.g. $S_{1} \rightarrow S_{4} \rightarrow S_{8}$ is a set of solution. Assuming that the user's requirement changes in $t$, so we need to adjust the manufacturing task $T_{2}$ that is in the state of execution, which causing the service $S_{4}$ unable to meet the requirement and the service needs to be reselected. It can be seen that traditional service composition methods need to replace multiple services simultaneously, and it is difficult to adapt to changes in dynamic environment, resulting in Qos degradation. 


\section{Models of manufacturing service-demand dynamic matching network}

\subsection{Models of manufacturing service network (MSN)}

Manufacturing service network (MSN) is the virtual network topology structure composed of a large number of service nodes and a variety of edges. Atomic manufacturing services (AMS) refer to fine-grained manufacturing services that can be invoked independently and are functionally indivisible, and is recorded as $S$. Manufacturing services can be either mapped to manufacturing resources with a single function, but also can be mapped to manufacturing resources with complex functions. The former is called fine- grained manufacturing service, that is, atomic manufacturing service. The latter, which we call coarse-grained manufacturing services, is denoted as $S^{C}$. We divided coarse-grained services $S^{C}$ into composite services made up of multiple atomic manufacturing services. Thus, there are only atomic manufacturing services in MSN. The manufacturing service dependency edge (MSDE) refers to the weighted directed edges based on the potential call relationship between atomic manufacturing services, which is denoted by $E^{S}=\left\{w_{i j}^{S}\right\}$, in which $w_{i j}^{S}=1$ indicates that there is an execution sequence relationship between manufacturing service $S_{i}$ and $S_{j}$.

Manufacturing service network (MSN) can be represented as directed acyclic network composed of AMS and MSDE. The basic service description is expanded as shown in formula.

Which,

$$
\text { Service }=\langle S-I D, B-\text { attri, } F-\text { attri, } U-\text { attri, } N-\text { attri, } M R P\rangle
$$

$F-$ attri $=\langle$ Ftype, input, output, constra int $s$, results $\cdots\rangle$

$U-$ attri $=\langle Q o S$, State,$S-$ time, $E-$ time, executionrecords $\cdots\rangle$

$N-$ attri $=\left\langle S-I D, E^{S}-\right.$ type $\left., E^{S}, W^{S} \cdots\right\rangle, M R P=\langle R-I D$, description, capability $\cdots\rangle$.

The parameters and notations in formula referred to the expanded manufacturing services description model are shown in tab. 1

Table1. Parameters and notations in manufacturing service description.

\begin{tabular}{|c|l|l|l|}
\hline $\begin{array}{l}\text { Notatio } \\
\mathrm{ns}\end{array}$ & Explanation & $\begin{array}{l}\text { Notation } \\
\mathrm{s}\end{array}$ & Explanation \\
\hline$S-I D$ & $\begin{array}{l}\text { Identification of manufacturing } \\
\text { service }\end{array}$ & $B-$ attri & $\begin{array}{l}\text { Basic attributes of manufacturing } \\
\text { service }\end{array}$ \\
\hline$F-$ Attr & $\begin{array}{l}\text { Functional attributes of } \\
\text { manufacturing service }\end{array}$ & $U-$ attri & $\begin{array}{l}\text { Using attributes of manufacturing } \\
\text { service }\end{array}$ \\
\hline -attri & $\begin{array}{l}\text { Network-related attributes of } \\
\text { manufacturing service }\end{array}$ & MRP & $\begin{array}{l}\text { Manufacturing resource mapped } \\
\text { to the service }\end{array}$ \\
\hline Ftype & $\begin{array}{l}\text { Type of functions of } \\
\text { manufacturing service }\end{array}$ & $\begin{array}{l}\text { End time of manufacturing } \\
\text { service execution }\end{array}$ & $\begin{array}{l}\text { Start time of manufacturing } \\
\text { service execution }\end{array}$ \\
\hline$E^{S}-t y p$ & Type of correlation edges in MSN & $E^{S}$ & $\begin{array}{l}\text { Identification of manufacturing } \\
\text { resource mapped to the service }\end{array}$ \\
\hline
\end{tabular}




\begin{tabular}{|l|l|l|l|}
\hline$W^{S}$ & $\begin{array}{l}\text { Weight of correlation edges in } \\
\text { MSN }\end{array}$ & & \\
\hline
\end{tabular}

The state of manufacturing services $S_{\text {status }}$ can be divided into two categories: not being invoked $\left(S_{\text {status }}=0\right)$ and being invoked $\left(S_{\text {status }}=1\right)$.

\subsection{Models of manufacturing task network (MTN)}

Similar to MSN, manufacturing task network (MTN) is also a virtual network topology structure composed of a large number of manufacturing task nodes and a variety of correlation edges among those task nodes. The cloud manufacturing platform can be decomposed into a series of atomic manufacturing tasks (AMT) with sequential relations through a certain strategy. AMT is an indivisible fine-grained manufacturing task, which can be executed by calling an atomic manufacturing service, and is recorded as $T$. The manufacturing task Executive Edge (MTEE) indicates the execution sequence relationship between atomic manufacturing tasks. It is obtained by the decomposition of coarse-grained manufacturing task $T^{c}$. MTEE is a weighted directed edge, which is recorded as $E^{T}=\left\{w_{i j}^{T}\right\}$, in which $w_{i j}^{T}=1$ indicates that there is an execution sequence relationship between manufacturing tasks $T_{i}$ and $T_{j}$. The basic executive relations of manufacturing tasks can be divided into 4 structures: order, concurrency, selection and circulation. Similarly, the description of manufacturing tasks oriented to the modelling of MTN is expanded as shown in formula.

$$
\text { Task }=\langle T-I D, B \text { inf, } F \text { inf, } E \text { inf, } N \text { inf, } \cdots\rangle
$$

Which, Binf $=\langle$ object, feature, material, comsumer, address, $\cdots\rangle$

$F$ inf $=\langle$ Ftype, precondition, effect, constraint $s, \cdots\rangle, N$ inf $=\left\langle T-I D, E^{T}-\right.$ type $\left., E^{T}, W^{T}\right\rangle$

The parameters and notations in formula referred to the expanded manufacturing task description model are shown in tab.2

Table 2. Parameters and notations in manufacturing task description.

\begin{tabular}{|l|l|l|l|}
\hline $\begin{array}{l}\text { Notation } \\
\mathrm{s}\end{array}$ & Explanation & $\begin{array}{l}\text { Notation } \\
\mathrm{s}\end{array}$ & Explanation \\
\hline$T-I D$ & $\begin{array}{l}\text { Identification of manufacturing } \\
\text { task }\end{array}$ & $B$ inf & $\begin{array}{l}\text { Basic information of manufacturing } \\
\text { task }\end{array}$ \\
\hline$F$ inf & $\begin{array}{l}\text { Functional information of } \\
\text { manufacturing task }\end{array}$ & $E$ inf & $\begin{array}{l}\text { Evaluation information of } \\
\text { manufacturing task }\end{array}$ \\
\hline$N$ inf & $\begin{array}{l}\text { Network-related information of } \\
\text { manufacturing task }\end{array}$ & $E^{T}-$ type & Type of correlation edges in MTN \\
\hline$E^{T}$ & $\begin{array}{l}\text { Judgment matrix of correlation } \\
\text { edges in MTN }\end{array}$ & $W^{T}$ & Weight of correlation edges in MTN \\
\hline
\end{tabular}

At different times, atomic manufacturing tasks are in different execution states $T_{\text {status }}$, and they are divided into four states: already execution $\left(T_{\text {status }}=0\right)$, prepare execution $\left(T_{\text {status }}=1\right)$, being execution $\left(T_{\text {status }}=3\right)$, and not executed $\left(T_{\text {status }}=4\right)$.

\subsection{Modeling of dynamic matching network}

\subsubsection{Model description}


Based on the models of MSN and MTN described in sections 3.1 and 3.2, the key point to construct the model of dynamic matching network (DMM), is to work out the existence of inter-network dynamic matching edge (INDME) which connect service nodes and task nodes. Inter-network dynamic matching edge indicates the dynamic matching relationship between atomic manufacturing task $T$ and atomic manufacturing service, which are defined as formula $E^{T S}=\left\{w_{i j}^{T S}\right\}$. When $w_{i j}^{T S}=1$, it is indicates that the manufacturing task $T_{i}$ and the manufacturing service $S_{i}$ are in a dynamic binding state. There is a dynamic matching edge between $T_{i}$ and $S_{i}$. When the manufacturing task $T_{i}$ is completed, the matching edge disappears. The time varying characteristics of the network are realized.

Dynamic matching network is a double-layer network formed by the dynamic matching relationship between manufacturing task network and manufacturing service network. It is recorded as $N e t^{T S}=\left\{N e t^{T}, N e t^{S}, E^{T S}\right\}$.

\subsubsection{Dynamic scheduling of manufacturing services}

QOS is an important evaluation standard for dynamic scheduling of manufacturing services. Due to the diversity of QoS indicators of manufacturing services, such as response time, cost, reliability, and so on, there are great differences between units and ranges. Therefore, it is usually necessary to normalize the QoS value, as shown in formula (3).

$$
U\left(S_{j i}\right)=\sum_{k=1}^{r} \frac{q_{k}\left(S_{j i}\right)-Q_{j, k}^{\min }}{Q_{k}^{\max }-Q_{k}^{\min }} \cdot w_{k}
$$

in which, $w_{k}$ is the weight of each attribute value of the QoS, which indicate the degree of preference for each attribute of a user. $Q_{j, k}^{\max }$ represents the maximum $k$ dimension attribute of QoS in all manufacturing service communities $C_{j}$. Manufacturing service community refers to a group of service classes that have the same or similar function but different QoS, which can be clustered according to the functional similarity between the services, $C^{S}=\left\{S_{1}^{C}, S_{2}^{C}, \cdots, S_{n}^{C}\right\}$, in which $n$ represents different atomic manufacturing services. $Q_{j, k}^{\min }$ represents the minimum $k$ dimension attribute of QoS in all manufacturing service communities $C_{j} \cdot q_{k}\left(S_{j i}\right)$ represents the $\mathrm{K}$ dimension attribute value of Qos in the candidate service $S_{j i}$.

Dynamic scheduling of manufacturing services has two optimization objectives. Firstly, from the perspective of users, cloud manufacturing platform needs to provide users with QoS optimal manufacturing services or service composition. Secondly, from the perspective of cloud manufacturing platform management, in a multi-user environment, how to realized the load balancing of various manufacturing service nodes through reasonable scheduling of resources and optimized the load queue is the other objectives. Suppose that the number of manufacturing service community that need to be invoked in the current manufacturing process is $N$, each manufacturing service community contains services number are $n$. We can model the dynamic scheduling of manufacturing services as formula (4). 


$$
\left\{\begin{array}{c}
\operatorname{ObjectMin}\left(W_{1} \cdot \sum_{j=1}^{N} \sum_{i=1}^{n} U\left(S_{j i}\right)\right)+W_{2} \cdot \sum_{j=1}^{N} \sum_{i=1}^{n} L\left(S_{j i}\right) \cdot x_{j i} \\
\text { Subjectto } \sum_{j=1}^{N} \sum_{i=1}^{n} q_{k}\left(S_{j i}\right) \cdot x_{j i} \leq \text { Cons }_{k}, 1 \leq k \leq r \\
\sum_{i=1}^{n} x_{j i}=1, x_{j i}=\{0,1\}
\end{array}\right.
$$

in which, $r$ represents the number of QoS properties of the service, $k$ represents the $k$ dimension property, $U\left(S_{j i}\right)$ represents the utility function value of the manufacturing service $S_{j i}$, which can be concluded by formula (5). $L\left(S_{j i}\right)$ represents the current load of the manufacturing service $S_{j i}$, Cons ${ }_{k}$ represents the constraint value of the $k$ dimension property. $x_{j i}$ is a decision variable, $x_{j i}=1$ indicates that the manufacturing service $S_{j i}$ is selected, in contrast $x_{j i}=0$ indicates that the manufacturing service $S_{j i}$ is not selected. Both $W_{1}$ and $W_{2}$ are both weights, and $W_{1}+W_{2}=1$.

Next, we will use shortest path routing strategy of complex network to solve the above models. The globally optimal service composition can be transformed to search for a least cost service composition path from the manufacturing service network, as shown in algorithm 1 .

Algorithm 1 Dynamic scheduling algorithm for manufacturing services

Input: $T_{\text {status }}$, Cons ${ }_{n-m}$, Service composition cost matrix MatrixC .

Output: $S_{j i}$.

1. $T=\operatorname{getExetask}\left(T_{\text {status }}\right)$

2. $C^{S}=\operatorname{getComunity}(T)$

3. FOR $j=m$ to $n$ do

4. len $=\operatorname{getLength}\left(C_{j}\right)$

5. FOR $i=1$ to len do

6. $\quad L_{j i}=\operatorname{getLoad}\left(S_{j i}\right)$

7. $\operatorname{QoS}_{j i}=$ updateQoS $\left(L_{j i}\right)$

8. END FOR

9. END FOR

10. MatrixC $=$ updateMatrix $\left(L_{j i}, Q o S_{j i}\right)$

11. MinPath $=\min$ Path $\left(\right.$ MatrixC, Cons $\left._{n-m}\right)$

12. $S C \leftarrow S C[1]$

13. Return $S_{j i}$

\section{Conclusions}

As the common issue to be addressed in advanced manufacturing systems, the supplydemand matching of manufacturing service in service-oriented manufacturing system is becoming to the decisive factor for its application and operation. Especially under the current socialization and cloud environment, there are different kinds of supply-demand 
matching problems considering the uncertainty and dynamics in service-oriented manufacturing systems. The theory of complex network provides an alternative method to analyse and address these problems.

From the perspective of complex network, this paper studies the dynamic management of manufacturing tasks and manufacturing services in cloud manufacturing systems. A dynamic matching network theory model has been built. The model takes the load and dynamic QoS as the optimization objectives, and transforms the optimal manufacturing service composition problem into the shortest path search in the manufacturing service network, so as to realize the dynamic scheduling of the manufacturing service.

Further research will improve the reliability of QoS's other indicators, such as the relationship between reliability and load, to improve the generality of the dynamic QoS model. Furthermore, we will study the impact of statistical properties of the service network on the service composition, such as the degree of nodes, and so on.

\section{Acknowledgments}

This work was supported by the National Natural Science Foundation under Grant number 71690233 and 71571185.

\section{References}

1. Baines, T., Bigdeli, A. Z. and Bustinza, O. Servitization: Revisiting the state-of-the-art and research priorities. International Journal of Operations\& Production Management, 2017, 37(2), pp 54-567.

2. F Tao., L. Zhang, Y. K. Liu, Y. Cheng etc. Manufactruing service management in cloud manufacturing: Overview and future research directions, Journal of manufacture science engineering-transaction, 2015,(4), pp:09-12.

3. Moon C, Lee $\mathrm{Y}$, Jeong $\mathrm{C}$, etc. Integrated process planning and scheduling in a supply chain. Computers\&Industrial Engineering, 2008, 54(4), pp. 1048-1061.

4. Zeng L, Benatallah B, Ngu AH, etc. QoS-aware middleware for Web services compositon. IEEE Transactions on Software Engineering, 2004,30(5), pp. 311-327.

5. Dai Y, Yang L, Zhang B. QoS-driven self-healing web service composition based on performance prediction. Journal of Computer Science and Technology, 2009, 24(2), pp. 250-261.

6. M. Dotoli, M.P. Fanti. C.Meloni, M.C. Zhou. Design and optimization of integrated Esupply chain for agile and environmentally conscious manufacturing. IEEE Transaction systems manufacturing, 2006, 36(1), pp. 62-75.

7. S.X. Huang, S. Zeng, Y.S. Fan. Optimal service selection and composition for serviceoriented manufacturing network. International Journal Computer integrate manufacture, 2011, 24(5), pp. 416-430.

8. F.Tao, D. Zhao, Y.F. Hu etc. Resource service composition and its optimal-selection based on particle swarm optimization in manufacturing grid system, IEEE Transactions and information, 2008, 4(4), pp. 315-327. 